\title{
Special feature: the seventh China-Japan-Korea joint conference on numerical mathematics (CJK2018)
}

\author{
Norikazu Saito ${ }^{1} \cdot$ Dongwoo Sheen ${ }^{2} \cdot$ Ziqing Xie $^{3}$ \\ Published online: 3 September 2019 \\ (c) The JJIAM Publishing Committee and Springer Japan KK, part of Springer Nature 2019
}

This special feature to be published in the present issue of the Japan Journal of Industrial and Applied Mathematics includes eight articles that were presented as papers at the Seventh China-Japan-Korea Joint Conference on Numerical Mathematics (CJK2018), which was held during August 20-25, 2018, at the Shiinoki Cultural Complex in Kanazawa City, Japan.

The series of CJK Conferences have been held every 2 years, alternately hosted by Japan, China, and Korea. Japan served as the host country for CJK2018. The organizing committee of each of the three countries respectively recommended seven invited speakers. The 21 invited lectures were the core of the program. In addition, contributed lectures were scheduled. This time, ten speakers, one each from China and Korea and eight from Japan, delivered their contributed lectures at the conference.

Leading the exchanges of numerical analysis researchers among Japan, China, and Korea over the years, CJK conferences have contributed greatly to improving the quality of research in the countries. As a result of those efforts, the global presence of these three countries is expanding considerably in the field of numerical analysis.

We would like to extend our gratitude again to Professor Hisashi Okamoto, Editorin-Chief, who granted us this opportunity to present some results of CJK2018 in the Japan Journal of Industrial and Applied Mathematics.

August 12, 2019

Norikazu Saito

norikazu@g.ecc.u-tokyo.ac.jp

Dongwoo Sheen

sheen@snu.ac.kr

Ziqing Xie

ziqingxie@hunnu.edu.cn

1 Graduate School of Mathematical Sciences, The University of Tokyo, Komaba 3-8-1, Meguro, Tokyo 153-8914, Japan

2 Department of Mathematics, Seoul National University, Seoul 151-747, South Korea

3 School of Mathematics and Statistics, Hunan Normal University, Changsha 410081, China 


\section{Norikazu Saito}

Dongwoo Sheen

Ziqing Xie

Publisher's Note Springer Nature remains neutral with regard to jurisdictional claims in published maps and institutional affiliations. 